\title{
Electromagnetic Processes in PANDA
}

\author{
Manuel Zambrana ${ }^{* \dagger}$ \\ Institut für Kernphysik, University of Mainz, Becher Weg 45, 55099, Mainz, Germany \\ E-mail: zambrana@kph.uni-mainz.de
}

This document reports on the feasibilty of measuring nucleon structure observables with the PANDA detector at the future FAIR facility. The future measurement of the time-like form factors of the proton using the reaction $\bar{p} p \rightarrow e^{+} e^{-}$with PANDA is discussed in detail, including background suppression and signal reconstruction efficiency. Results of the simulations show that an unprecedent precision in the measurements can be achieved with PANDA. The possibility of measuring the form factors in the unphysical region, below threshold, is also discussed. In addition to the measurement of the form factors, the first feasibility studies to access the proton to pion Transition Distribution Amplitudes using the channel $\bar{p} p \rightarrow e^{+} e^{-} \pi^{0}$ in the regime of QCD collinear factorization are also presented.

Photon 2013,

20-24 May 2013

Paris, France

* Speaker.

$\dagger$ on behalf of the PANDA Collaboration 


\section{Introduction}

Quantum Chromodynamics (QCD) is, as far as presently known, the correct theory of the strong interaction. It is responsible for the formation of hadrons, non-perturbative particle states which are interpreted in terms of interacting quarks and gluons, the fundamental degrees of freedom of the theory. The quark electric charge then endows the hadron with an electromagnetic structure, properly parametrized by the matrix element of the electromagnetic current between hadron states $\left\langle p^{\prime}\left|j_{\mu}(0)\right| p\right\rangle$. Parity invariance, Gordon identities and current conservation (see, for instance, [1], chapter 12, section 12.1) allow to express the matrix element in terms of two scalar functions of the four momentum transfer squared, the Pauli and Dirac form factors $F_{1}\left(q^{2}\right)$ and $F_{2}\left(q^{2}\right)$ :

$$
\left\langle p^{\prime}\left|j_{\mu}(0)\right| p\right\rangle=\bar{u}\left(p^{\prime}\right)\left\{F_{1}\left(q^{2}\right) \gamma_{\mu}+F_{2}\left(q^{2}\right) \frac{1}{4 M}\left[\hat{q}, \gamma_{\mu}\right]\right\} u(p), \quad \hat{q} \equiv q_{\nu} \gamma_{\nu},
$$

where $q=p^{\prime}-p$ and $M$ is the mass of the hadron. The electric and magnetic form factors $G_{E}$ and $G_{M}$ (also called Sachs form factors), are then defined as the linear combinations $G_{E}=F_{1}+\tau F_{2}$ and $G_{M}=F_{1}+F_{2}$, with $\tau=4 M^{2} / q^{2}$. In the space-like region $\left(q^{2}<0\right)$, the form factors are real functions of the four momentum transfer, whereas in the time-like region $\left(q^{2}>0\right)$, they acquire non-zero imaginary part. By continuation of $q^{2}$ to imaginary values, the form factors become analytical functions of their argument. The form factors encode all the electromagnetic structure of the hadron brought by the strong interaction. In the Breit frame, space-like $G_{E}$ and $G_{M}$ are interpreted as the Fourier transform of the spatial charge and magnetization distribution, with the slope at $q^{2}=0$ giving the charge and magnetization radius of the hadron. The measurement of the form factors yields therefore a direct observation of the electromagnetic structure of the nucleon, providing a test of the QCD predictions brought by non-perturbative approaches, like lattice field theory or chiral perturbation theory.

The electromagnetic probe is then an excellent tool to investigate the structure of the nucleon. In both electron scattering $e^{ \pm} p \rightarrow e^{ \pm} p$ and the corresponding annihilation cross process $\bar{p} p \rightarrow e^{+} e^{-}$ (see Figure 1), the scattering amplitude is proportional to the matrix element (1.1). Scattering processes, with $q^{2}<0$, allow to access the space-like form factors. Annihilation processes allow to access the time-like form factors above the kinematic threshold $q^{2}>4 M^{2}$. The gap $0<q^{2}<4 M^{2}$ is not directly accessible by neither scattering or annihilation experiments and for this reason it is called the "unphysical region".

Early investigations on the form factors of the nucleon date from the late fifties. However, it continues being a hot topic in hadron physics today, with many open questions not yet answered. Just to mention a few, the matrix element (1.1), highly explored in the space-like region, is still much less known in the time-like region; the extraction of the proton radius using muonic hydrogen disagrees significantly with that extracted from electronic hydrogen or elastic electron-proton scattering [2]; Rosenbluth separation techniques and polarization data yield incompatible results for the space-like form factors of the nucleon; the structure of the unphysical region, not directly accessible by the experiment, and its resonance content is of crucial importance for the implications in dispersive analysis, connecting the values of the form factors in the space-like and time-like regions.

The current experimental data base includes many high precision measurements of the electromagnetic form factors in the space-like region, using either Rosenbluth separation or polariza- 
tion transfer techniques. On the other hand, the time-like region remains poorly explored, with just low precision measurements from total cross sections and a few angular distribution measurements, usually done under the assumption $\left|G_{E}\right|=\left|G_{M}\right|$. For a comprehensive review of the world data base, we refer to [3]. In the time-like region, the data base includes PS170 [4] and BABAR measurements [5]. The detailed exploration of time-like region will be a prominent issue in the experimental program of the future PANDA experiment.
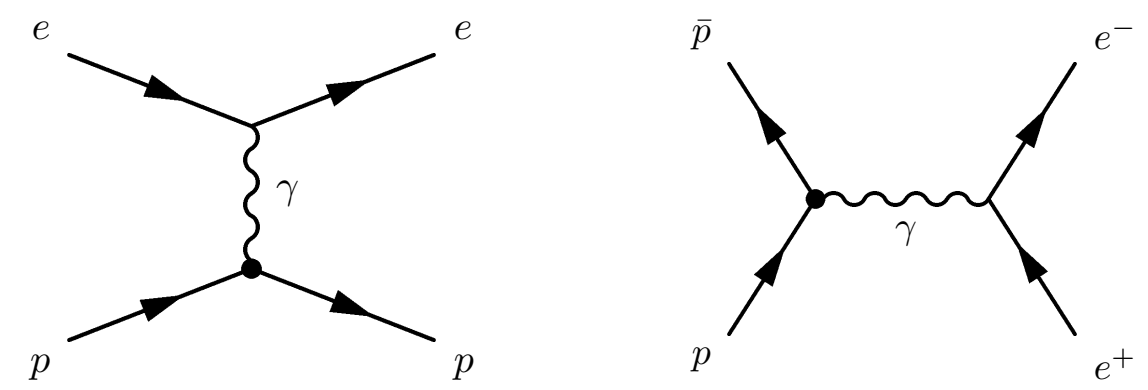

Figure 1: Feynman diagrams for elastic electron proton scattering (left) and proton anti-proton annihilation (right) at the lowest order in the coupling. The electromagnetic form factors enter at the photon nucleon vertex.

\section{The future PANDA experiment}

PANDA (antiProton ANnihilations at DArmstadt) [6] will be one of the experiments at the future FAIR/GSI facility (Facility for Antiproton and Ion Research) [7]. The PANDA experiment is a multipurpose, high energy physics experiment runned by a collaboration of about 400 physicists from 14 countries. The wide scientific program extends from hadron spectroscopy (up to the charm sector), hadron structure and non-perturbative dynamics to hypernuclei physics. Investigations of the hadron structure include a dedicated program to measure the time-like electromagnetic form factors of the proton, mainly through the annihilation process $\bar{p} p \rightarrow e^{+} e^{-}$, with unprecedent precision.

A High Energy Storage Ring (HESR), will provide a high quality antiproton beam of momentum between 1.5 to $15 \mathrm{GeV}$. The conception of the detector, the read out and the data acquisition system keeps similarity with other recently built detectors, such as ATLAS, CMS, COMPASS and BABAR. However, the high expected rate of $2 \cdot 10^{7}$ interactions/s and the multipurpose character of the detector demand unique detection capabilities in PANDA, including geometrical acceptance of almost $4 \pi$, energy and momentum resolution at a few percent level, good particle identification (PID), fast data acquisition and high radiation hardness. The detector consists of a barrel spectrometer and a forward spectrometer. In the barrel spectrometer, which will be mainly used for the measurement of the electromagnetic form factors, it combines a solenoidal magnetic field with micro-vertex and gas tracking detectors with a DIRC-detector and a high resolution fast electromagnetic PWO-calorimeter. The particle identification capabilities of PANDA have been studied in detailed simulations. Sets of particles of different species have been generated at given momenta and polar angle. The probabilities for identification of a given particle as electron, muon, pion, 
kaon, or proton are then calculated first for each individual subdetector using the relevant kinematic variables in each case. These include the ratio $E / p$ of the measured energy deposit to the reconstructed momentum and shower shapes in the electromagnetic calorimeter, the Cerenkov angle in the DIRC detector, the ratio of energy loss to path lengh $d E / d x$ in the STT and microvertex detector and information from the muon chambers. Figure 2 shows, as an example, how the distribution of $E / p$ in the calorimeter and shower shapes can be used to discriminate between different particle species, electron/pion separation in particular. From the individual subdetector likelihoods, a global PID likelihood is then calculated. Depending on the signal and background channels, the cuts can be adjusted in order to ensure the required purity while keeping the signal efficiency as high as possible.
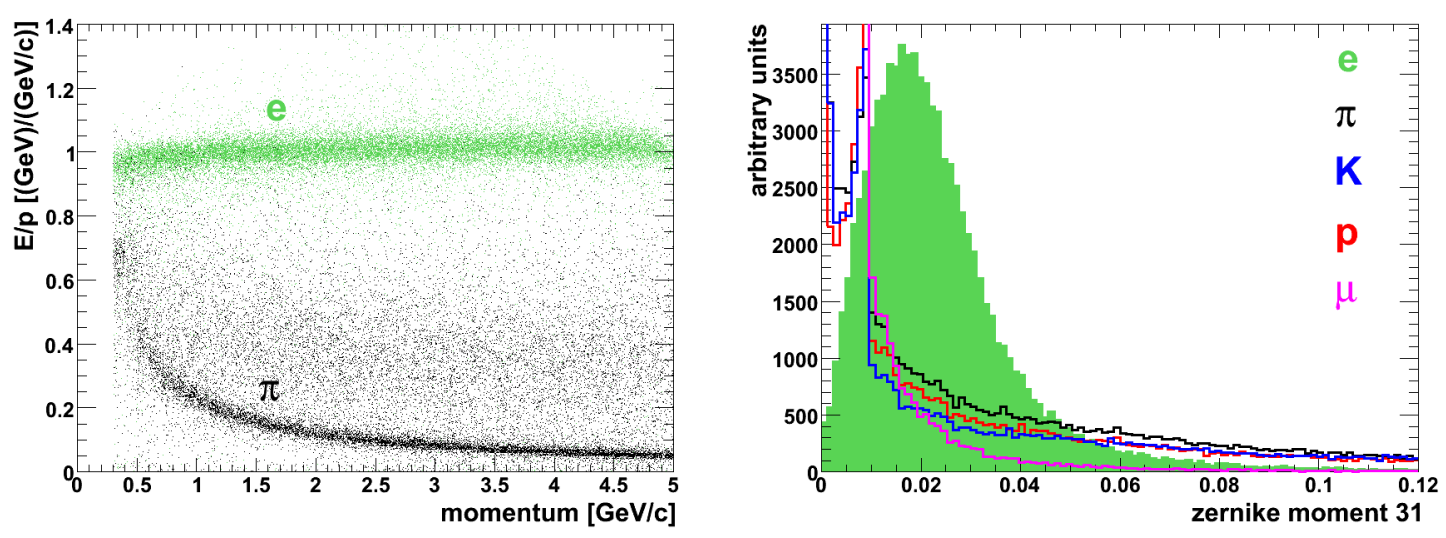

Figure 2: The ratio $E / p$ of the measured energy deposit to the reconstructed momentum for electrons and pions in the electromagnetic calorimeter (left). Shower shapes for different particle species in the electromagnetic calorimeter (right).

\section{Measurement of the time-like electromagnetic form factors with $\bar{p} p \rightarrow e^{+} e^{-}$}

The differential cross section for the annihilation process $\bar{p} p \rightarrow e^{+} e^{-}$was first obtained in Ref [8]. At the lowest order in the coupling, the proccess occurs through the so called one photon exchange (OPE) mechanism, that is, the annihilation of the initial hadron state $\bar{p} p$ into a virtual photon $\gamma^{*}$ which then decays to the lepton pair $e^{+} e^{-}$observed in the final state. The only diagram which contributes to the tree-level scattering amplitude was shown in Figure 1 (left). At this order and neglecting the mass of the electron, the differential cross section at the $\bar{p} p$ center of mass energy squared $s$ is given by:

$$
\frac{d \sigma}{d \cos \theta^{*}}=\frac{\pi \alpha^{2}}{2 s} \frac{1}{\beta}\left\{\left(1+\cos ^{2} \theta^{*}\right)\left|G_{M}\right|^{2}+\frac{1}{\tau}\left(1-\cos ^{2} \theta^{*}\right)\left|G_{E}\right|^{2}\right\},
$$

where $M$ is the mass of the proton, $\tau=q^{2} / 4 M^{2}$ with $q^{2}=s, \beta=\sqrt{1-4 M^{2} / s}$ and $\theta^{*}$ is the angle between the electron and the antiproton in the $\bar{p} p$ center of mass system. The moduli of the form factors $\left|G_{E}\right|$ and $\left|G_{M}\right|$ can be the extracted independently from the angular distribution 
of the $\bar{p} p \rightarrow e^{+} e^{-}$events by fitting the data with the function given by Eq. (3.1), where $\left|G_{E}\right|$ and $\left|G_{M}\right|$ play the role of independent parameters. A good luminosity measurement is required for a separated extraction, otherwise the absolute normalization of the cross section remains unknown and only the ratio $\left|G_{E}\right| /\left|G_{M}\right|$ is accessible. One experimental difficulty in the determination of the time-like form factors using the channel $\bar{p} p \rightarrow e^{+} e^{-}$arises from the fact that the cross sections ratio $\sigma\left(\bar{p} p \rightarrow \pi^{+} \pi^{-}\right) / \sigma\left(\bar{p} p \rightarrow e^{+} e^{-}\right)$is of the order of $10^{6}[9,10,11,12]$. Two pion production $\bar{p} p \rightarrow \pi^{+} \pi^{-}$, with a final state containing the same number of particles and charge signature as $\bar{p} p \rightarrow e^{+} e^{-}$, becomes therefore the most severe background channel in the measurement of the form factors. If we aim for a $1 \%$ pollution of the $\bar{p} p \rightarrow e^{+} e^{-}$signal events, a misidentification probability for $\bar{p} p \rightarrow \pi^{+} \pi^{-}$of only $10^{-8}$ has to be achieved. PID then becomes crucial.

The feasibility of measuring the time-like form factors of the proton $\left|G_{E}\right|$ and $\left|G_{M}\right|$ with the PANDA detector has been studied in an extended range of antiproton beam momentum running from $1.7 \mathrm{GeV}$ to $13.4 \mathrm{GeV}[13,14]$. The suppression of the most severe background channel, $\bar{p} p \rightarrow$ $\pi^{+} \pi^{-}$has been studied first. To account for background production, the $\bar{p} p \rightarrow \pi^{+} \pi^{-}$cross section has been modelled differently in each energy range according to the existing data and/or theoretical predictions available in each case. At low energies, up to $s<6 \mathrm{GeV}^{2}$, the parametrization was taken to be a Legendre polynomial fit to the existing data [9]. At higher energies, $s>9 \mathrm{GeV}^{2}$, the recent theoretical predictions [15] based on Regge models were used. Statistical samples containing $10^{8}$ $\pi^{+} \pi^{-}$events at $s=8.2 \mathrm{GeV}^{2}$ and $s=12.9 \mathrm{GeV}^{2}$, and $2 \cdot 10^{8}$ events at $s=16.7 \mathrm{GeV}^{2}$ were used to study background suppression. Using particle identification capabilities of the central gas tracking detector, the DIRC-detector and the electromagnetic calorimeter in combination with a kinematical fit to the final state charged tracks, no $\pi^{+} \pi^{-}$events were misidentified as $e^{+} e^{-}$events out of $10^{8}$ and $2 \cdot 10^{8}$ for the three energies studied. Simulations show, therefore, that event selection in PANDA will yield a suppression factor of $10^{8}$ for pions, ensuring that the pollution of the $\bar{p} p \rightarrow e^{+} e^{-}$signal events will remain below $1 \%$, while keeping the signal reconstruction efficiency at the level of $40 \%$ on the average, as shown in Figure 3.
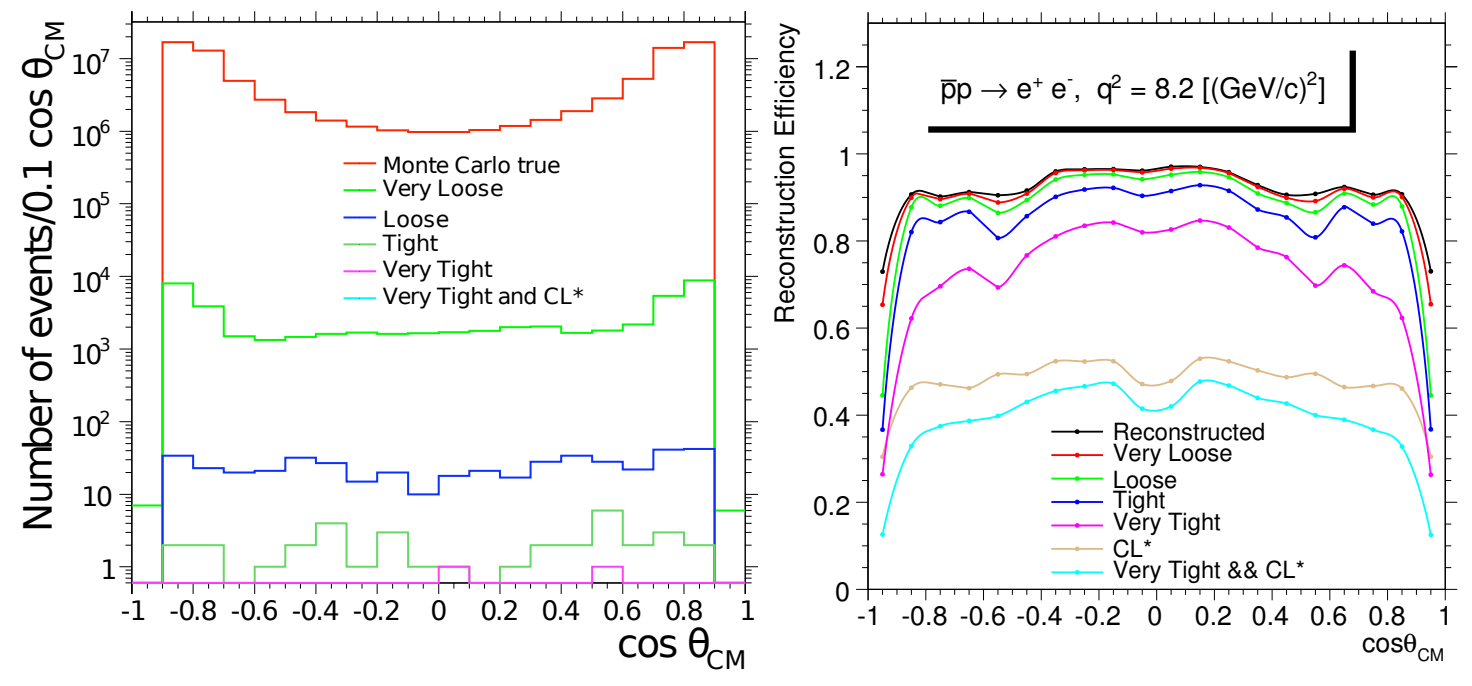

Figure 3: Suppresion of the $\bar{p} p \rightarrow \pi^{+} \pi^{-}$background events (left) and reconstruction efficiency for the $\bar{p} p \rightarrow e^{+} e^{-}$signal events (right) after event selection. 
In addition, full simulations on the angular distribution of the $\bar{p} p \rightarrow e^{+} e^{-}$were done at several antiproton beam momenta and under three different hypothesis of the electric to magnetic form factor ratio $\left|G_{E}\right| /\left|G_{M}\right|=0,1,3$. In each case, the expected number of events corresponding to an integrated luminosity of $2 \mathrm{fb}^{-1}$ were simulated. After efficiency correction, the corresponding distributions were fitted using the (non-normalized) fit function given by Eq. (3.1) in order to extract a "measured" value for $\left|G_{E}\right| /\left|G_{M}\right|$. The quality of the data and their statistical accuracy shows that a measurement of the electromagnetic form factors with a precision of a few percent in the overlaping region with the data from BABAR, which display errors of the order of $40 \%$, will be possible with PANDA. The ratio of the electric to magnetic form factor will be measurable until $s \sim 14 \mathrm{GeV}^{2}$, with an error which compares to the existing data at much lower energies. An example is shown in Figure 4 below. Above this energy, it is still possible to extract an effective form factor from the total cross section measurement and test its asymptotic behaviour.
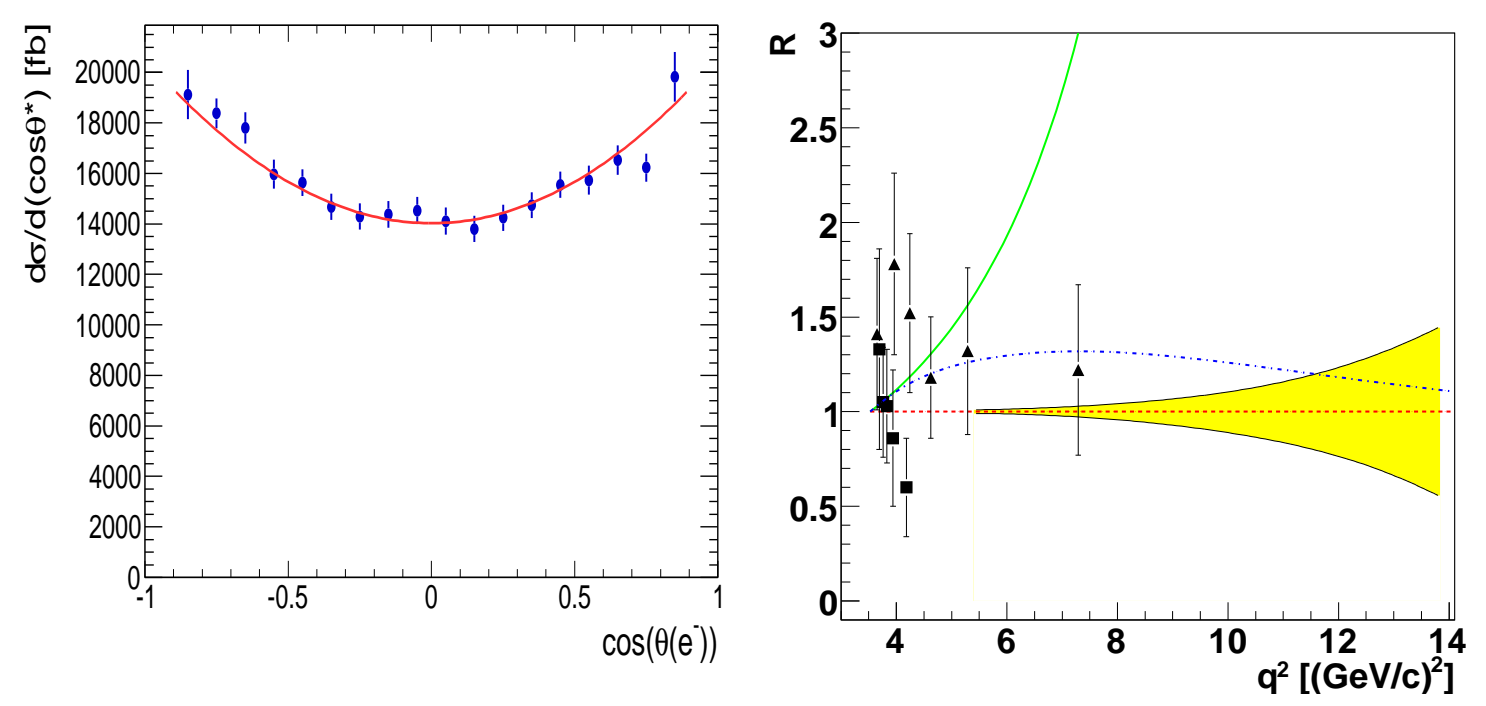

Figure 4: Angular distribution of signal events at $s=8.2 \mathrm{GeV}^{2}$ assuming the ratio $\left|G_{E}\right| /\left|G_{M}\right|=1$ using a statistical sample with integrated luminosity $2 \mathrm{fb}^{-1}$ (left). Expected statistical precision on the determination of the ratio $\left|G_{E}\right| /\left|G_{M}\right|$ (yellow band) assuming the true value $\left|G_{E}\right| /\left|G_{M}\right|=1$, as a function of $q^{2}$, compared to data from PS170 [4] (triangles) and BABAR [5] (squares) (right).

The extraction of the time-like form factors of the proton using electromagnetic processes is subjected to radiative corrections. So far, corrections which take into account only final state radiation were considered by using standard Monte Carlo programs [16]. Recent model-independent calculations [17] of the radiative corrections for the process $\bar{p} p \rightarrow e^{+} e^{-}$take into account both initial and final state radiation and their interference. The calculation shows that the size of the corrections may be of the order of a few percent, comparable to the precision of the PANDA measurements. The theoretical predictions can then be used to correct the measured cross sections to bring them to the Born level, as needed for the extraction of the form factors. The development of the event generators and their interface to the PANDA simulation software is currently under consideration. 


\section{Time-like form factors below threshold}

The unphysical region $0<q^{2}<4 M^{2}$, with its resonance content, becomes of fundamental importance in the theory, particularly in dispersion relations conncecting space-like and time-like regions. However, it is not accessible with the process $\bar{p} p \rightarrow e^{+} e^{-}$. The production of a lepton pair in association with a neutral pion, i.e. $\bar{p} p \rightarrow e^{+} e^{-} \pi^{0}$ has been proposed as a window into the unphysical region $[18,19]$. The reaction proceeds in two steps: first, annihilation of proton and antiproton into a virtual photon and a neutral pion, i.e. $\bar{p} p \rightarrow \gamma^{*} \pi^{0}$, followed by the decay of the virtual photon into the dilepton pair, i.e. $\gamma^{*} \rightarrow e^{+} e^{-}$. As part of the total initial state four-momentum can be transferred to the neutral pion, the photon virtuality $q^{2}$ is not any more constrained to be above the threshold $q^{2}>4 M^{2}$ (as in $\bar{p} p \rightarrow e^{+} e^{-}$), so the kinematic region $4 m_{e}^{2}<q^{2}<4 M^{2}$ becomes, in principle, accessible. Phenomenological one-nucleon exchange models [20] as well as a Regge approach [21] have been recently proposed to describe the cross section of the process. The latter, in particular, has been tested by considering the limit of real photoproduction, i.e. $\bar{p} p \rightarrow \gamma \pi^{0}$, reproducing the existing data [22]. In addition to the potential access to the unphysical region, the analysis of the dilepton phase space in the $\gamma^{*}$ rest frame opens the possibility of measuring not only the form factor moduli, but also their phase difference. The modulation of the differential cross section in bins of the azimuthal coordinate $\Phi_{e^{+}} e^{-}$(the angle between leptonic and hadronic plane) gives access, through the interference terms $G_{E} G_{M}^{*}$ (and complex conjugate), to the form factors phase difference (see Figure 5). This is of particular importance because it does not require polarization of the antiproton target, usually complicated from the experimental point of view.
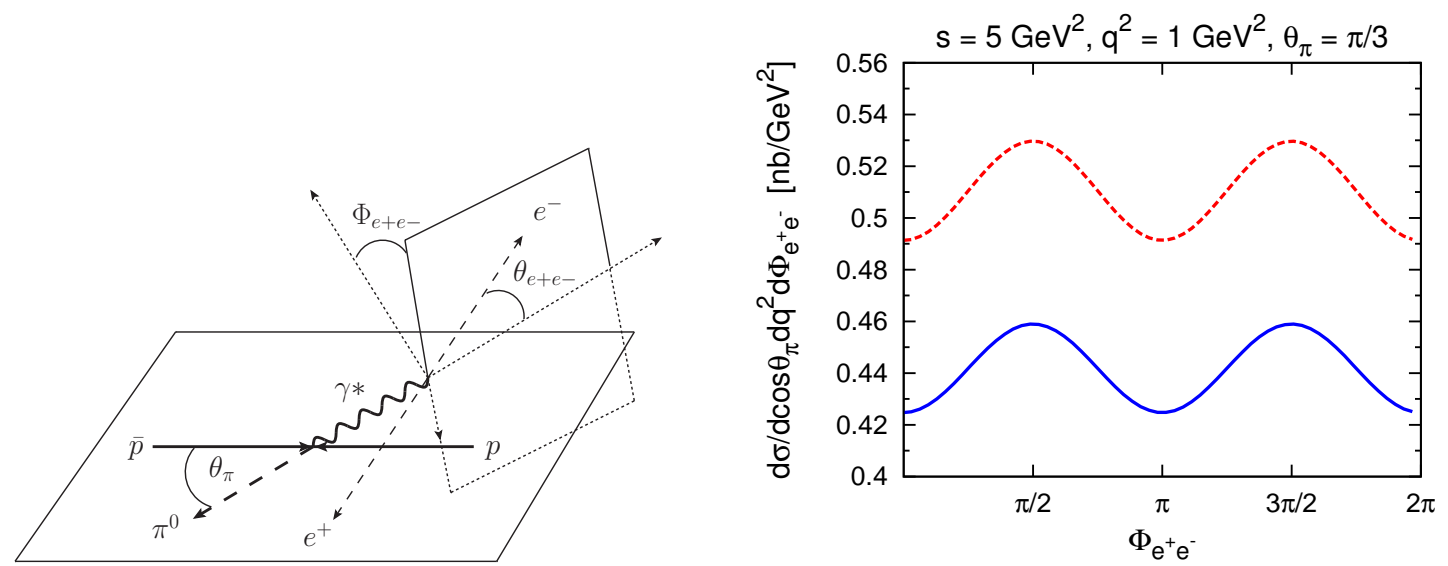

Figure 5: Kinematics of the $\bar{p} p \rightarrow e^{+} e^{-} \pi^{0}$ process (left). Differential cross section as a function of the angle between the hadronic and leptonic plane $\Phi_{e^{+} e^{-}}$(right). The red (dashed) line represents the contribution of the nucleon trajectory in the Regge theory description; the blue (solid) line represents the contribution of the nucleon and delta trajectories. The figures are taken fron Ref. [21]

Feasibility studies of measuring $\bar{p} p \rightarrow e^{+} e^{-} \pi^{0}$ have been performed [23] under the assumption of a one-nucleon-exchange production mechanism. The most severe background to this process is three pion production, i.e. $\bar{p} p \rightarrow \pi^{+} \pi^{-} \pi^{0}$, as it contains the same number of final state particles as the signal channel with identical charge signature. A first order model (including the $\rho$ and $f_{2}$ resonances) for the background channel was developed and constrained by using existing data [24]. 
Results of the simulation show that the background can be suppressed up to the level of $1 \%$ (or even less) pollution of the $\bar{p} p \rightarrow e^{+} e^{-} \pi^{0}$ signal events, while keeping the signal reconstruction efficiency at the level of a few percent to $30 \%$. The precision in the measurement of the ratio $\left|G_{E}\right| /\left|G_{M}\right|$ was found to be of the order of a few percent with the simulations. For the first time, the feasibility of extracting the (cosine) form factor phase difference $\varphi_{E}-\varphi_{M}$ was studied. The simulations show that the precision of $\cos \left(\varphi_{E}-\varphi_{M}\right)$ varies from $10 \%$ at $q^{2}=0.605 \pm 0.005 \mathrm{GeV}^{2}$ to $20 \%$ at $q^{2}=2.0 \pm 0.125 \mathrm{GeV}^{2}$.

\section{Experimental access to the Transition Distribution Amplitudes}

In addition to the determination of the time-like electromagnetic form factors, the PANDA experiment will be able to access more nucleon structure observables through hard reactions for which QCD factorization applies. This will offer new ways of looking on the hadron's interior to bring different perspectives to the problem of the hadron structure in terms of quarks and gluons, the fundamental degrees of freedom of the strong interaction. One of these channels is protonantiproton annhilation into a lepton pair with high invariant mass $q^{2}$ in association with a neutral pion of low transverse momentum. Under these conditions, the reaction $\bar{p} p \rightarrow e^{+} e^{-} \pi^{0}$ admits a factorized description in terms of antinucleon (or nucleon) Distribution Amplitudes (DAs) and nucleon to meson (or antinucleon to meson) Transition Distribution Amplitudes (TDAs) [25, 26]. The TDAs [27] then appear as universal non-perturbative objects describing the transition between two different particles and can be considered, in a sense, as an extension of the Generalised Parton Distributions (GPDs) [28]. The TDAs are defined from the Fourier transform of a matrix element of a three-quark-light-cone operator between a proton and a meson state, with QCD evolution equations which follow from the renormalization group equations of the three-quark operator. The future measurement of the signal channel cross section with PANDA will provide a new test of perturbative QCD and open the possibility of accessing the proton to pion Transition Distribution Amplitudes.

The feasibility of accessing the proton/antiproton to pion TDAs with the PANDA detector through the measurement of the differential cross section for the signal channel $\bar{p} p \rightarrow e^{+} e^{-} \pi^{0}$ has been studied [14]. Detailed simulations on signal reconstruction efficiency as well as on rejection of the most severe background channel, i.e. $\bar{p} p \rightarrow \pi^{+} \pi^{-} \pi^{0}$, have been performed at the center of mass energy squared $s=5 \mathrm{GeV}^{2}$ and $s=10 \mathrm{GeV}^{2}$, in the kinematic region of four-momentum transfer $3.8<q^{2}<4.2 \mathrm{GeV}^{2}$ and $7<q^{2}<8 \mathrm{GeV}^{2}$, respectively, with $\left|\cos \theta_{\pi^{0}}>0.85\right|$. For the description of the signal channel, the early estimates presented in Ref. [25], with a forward neutral pion $\left(\cos \theta_{\pi^{0}}=1\right.$, or $t$-channel $)$ or with a backward neutral pion $\left(\cos \theta_{\pi^{0}}=-1\right.$, or $u$-channel $)$ were used as the input for the event generator. In the absence of data or theoretical predictions in the kinematic region of the measurement, three pion production $\bar{p} p \rightarrow \pi^{+} \pi^{-} \pi^{0}$ was treated as having the same angular distributions as those of the signal channel, and assuming, in addition, the cross sections ratio $\sigma\left(\bar{p} p \rightarrow \pi^{+} \pi^{-} \pi^{0}\right) / \sigma\left(\bar{p} p \rightarrow e^{+} e^{-} \pi^{0}\right)=10^{6}$. This represents, from the experimental side, the most unfavoured case, as background discrimination relies entirely on PID. Simulations show that PANDA particle identification capabilities in combination with kinematical constraints will allow a supression of the hadronic background $\bar{p} p \rightarrow \pi^{+} \pi^{-} \pi^{0}$ at the level of $10^{8}$ in all cases. In addition, the dedicated simulations using the expected statistics corresponding to the integrated 
luminosity of $2 \mathrm{fb}^{-1}$ show that the future measurement of the production differential cross section in bins of $q^{2}$ is feasible with PANDA, with average statistical uncertainties running from $16 \%$ to $18 \%$ at $s=5 \mathrm{GeV}^{2}$ and from $23 \%$ to $40 \%$ at $s=10 \mathrm{GeV}^{2}$. Results, as shown in Figure 6, open the possibility of accessing the Transition Distribution Amplitudes with the future PANDA experiment.
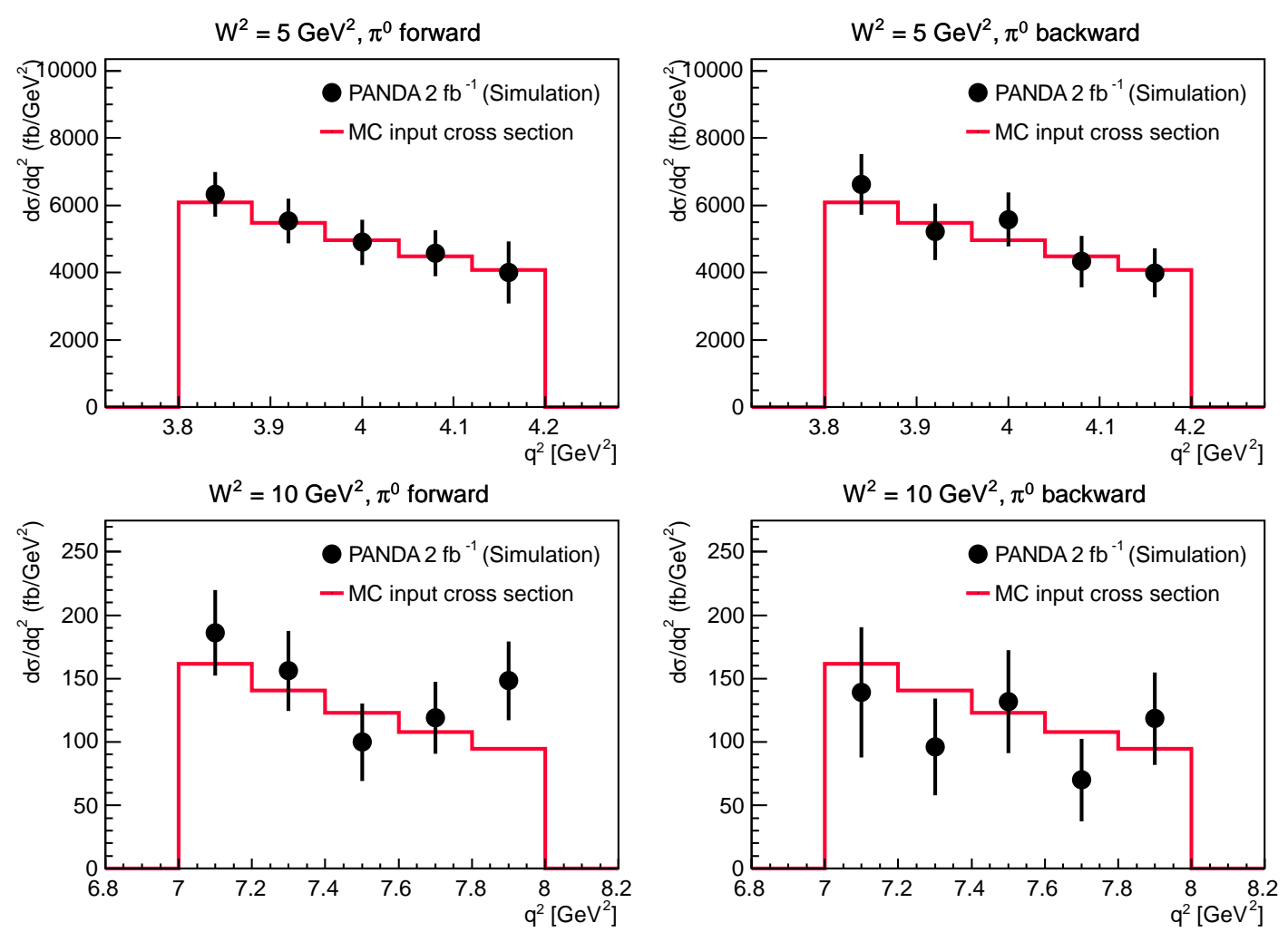

Figure 6: The "measured" $\bar{p} p \rightarrow e^{+} e^{-} \pi^{0}$ differential cross section with the simulation $\left(d \sigma / d q^{2}\right)$ in bins of $q^{2}$ with a statistical sample of integrated luminosity $\mathscr{L}=2 \mathrm{fb}^{-1}$, compared to the theoretical input in the Monte Carlo, for $s=5 \mathrm{GeV}^{2}$ and $s=10 \mathrm{GeV}^{2}$, in both the $t$ ( $\pi^{0}$ forward) and $u\left(\pi^{0}\right.$ backward) channel.

Acknowledgements This work has been supported by the Bundesministerium für Bildung und Forschung (BMBF), under grant number 05P12UMFP9. The speaker would like to thank the organizers of the Photon 2013 Conference for their invitation and for providing an exciting atmosphere for scientific discussion. We would like to express our gratitude to the PANDA Collaboration for their constant help and support. We would like to thank B. Pire, L. Szymanowski, K. Semenov and J.P. Lansberg for constructive and stimulating discussions and lots of explanations on the topic of the TDAs. Finally, we are very grateful to D. Djukanovic for his invaluable work in maintaining the dedicated HIMSTER cluster at Helmholtz-Institut-Mainz, where most of our simulations have been performed.

\section{References}

[1] G. F. Sterman, An Introduction to quantum field theory, Cambridge University Press, New York 1993. 
[2] R. Pohl, R. Gilman, G. A. Miller and K. Pachucki, Muonic hydrogen and the proton radius puzzle, arXiv:1301.0905 [physics.atom-ph].

[3] C. F. Perdrisat, V. Punjabi and M. Vanderhaeghen, Prog. Part. Nucl. Phys. 59, 694 (2007) [hep-ph/0612014].

[4] G. Bardin et al., Nucl. Phys. B 441, 3 (1994).

[5] BABAR Collaboration (B. Aubert et al.), Phys. Rev. D73, 012005 (2006).

[6] M. F. M. Lutz et al. [PANDA Collaboration], Physics Performance Report for PANDA: Strong Interaction Studies with Antiprotons, [arXiv:0903.3905 [hep-ex]].

[7] FAIR Baseline Technical Report, Technical Report, Gesellschaft für Schwerionenforschung (GSI) (2006).

[8] A. Zichichi et al., Nuovo Cim. 24, 170 (1962).

[9] E. Eisenhandler et al., Nucl. Phys. B 96, 109 (1975).

[10] T. Buran et al., Nucl. Phys. B 116, 51 (1976).

[11] T. Berglund et al., Nucl. Phys. B 137, 276 (1978).

[12] R. Dulude et al., Phys. Lett. 79B, 329 (1978).

[13] M. Sudol et al., Eur. Phys. J. A 44, 373-384 (2010).

[14] M. C. Mora Espí, Feasibility studies for accessing nucleon structure observables with the PANDA experiment at the future FAIR facility, PhD thesis, Mainz 2012.

[15] J. Van de Wiele and S. Ong, Eur. Phys. J. A 46, 291 (2010).

[16] E. Barberio and Z. Was, Comput. Phys. Commun. 79, 291 (1994).

[17] J. Van de Wiele and S. Ong, Eur. Phys. J. A 49, 18 (2013).

[18] M. P. Rekalo, Sov. J. Nucl. Phys. 1, 760.

[19] A. Z. Dubnickova et al., Z. Phys. C 70, 473 (1996).

[20] C. Adamuscin et al., Phys. Rev. C 75, 045205 (2007).

[21] J. Guttmann and M. Vanderhaeghen, Phys. Lett. B 719, 136 (2013).

[22] E760 Collaboration, Phys. Rev. D 56, 2509 (1997).

[23] J. Boucher, Feasibility studies of the $\bar{p} p \rightarrow e^{+} e^{-} \pi^{0}$ electromagnetic channel at PANDA, PhD thesis, Mainz 2011.

[24] T. C. Bacon et al., Phys. Rev. D 7, No. 3, 577 (1973).

[25] J. P. Lansberg, B. Pire and L. Szymanowski, Phys. Rev. D 76, 111502 (2007).

[26] J. P. Lansberg, B. Pire, K. Semenov-Tian-Shansky and L. Szymanowski, Phys. Rev. D 86, 114033 (2012).

[27] B. Pire and L. Szymanowski, Phys. Rev. D 71, 111501 (2005).

[28] L. L. Frankfurt et al., Phys. Rev. D 60, 014010 (1999). 